\title{
A Method for Improving Recognition of Lying Postures Using a Measured Signal Intensity of Respiration and Heartbeat by Flexible Tactile Sensor Sheet
}

\author{
Kazuya Matsuo \\ Department of Mechanical and Control Engineering, Kyushu Institute of \\ Technology, 1-1, Sensui, Tobata, Kitakyushu, Fukuoka, 804-8550, Japan \\ Toshiharu Mukai \\ Meijo University, Japan \\ Shijie Guo \\ Hebei University of Technology, China \\ E-mail:matsuo@cntl.kyutech.ac.jp
}

\begin{abstract}
We describe a method for improving recognition of lying postures using a measured signal ntensity of respiration and heartbeat. We can obtain respiration and heartbeat by means of using the body pressure measured at the suitable location determined by lying posture in the conventional method. Therefore, a recognition rate of lying postures and a measured signal intensity of respiration and heartbeat have positive correlation. In the experiments, we show that recognition of lying postures is improved by our method.
\end{abstract}

Keywords: Unconstrained measurement, Lying posture, Respiration, Heartbeat, ubber-based flexible tactile sensor sheet.

\section{Introduction}

Japan's population of people aged 65 and above increased by 7 million, that is, from 29 million to 36 million in the ten years between 2010 and 2020. Further, the aging rate is expected to increase from $23.0 \%$ to $29.1 \%$ [1], [2]. Seemingly, the number of people that require long-term care will substantially increase. However, the burden on individual care staff is expected to increase as there will be no corresponding increase in the number of care staff [3]. There is therefore an increasing need for the introduction of care robots in order to reduce the burden on care staff and further solve this problem.

The main areas of focus that have been chosen for the use of robot technology in care are transfer assistance, mobility support, toilet support, watching over dementia patients and bathing assistance [4]. Out of these, the ability to use sensors to watch over dementia patients would significantly help to reduce the burden on care staff. Moreover, there are many benefits of the ability to identify a patient's position in bed that are not limited to dementia patients. The patient positions

that must be identified include the patient being in or out of bed, sitting up or lying down, any danger of the patient falling out of bed or a patient lying on their back or on their front. Additionally, the ability to measure the depth or state of sleep is useful too. Increasing the quality of sleep by observing the state of sleep is effective for maintaining the patient's healthy sleep state.

The standard and reliable method for measuring sleep states is polysomnography (PSG) [5]. This technique measures brain waves, ECG, eye movement, chin muscle activity and pneumogram. Further, based on the R\&K method [6], the technique classifies sleep state into six categories namely wake, REM sleep and non-REM sleep stages 14. The technique is beneficial as it measures sleep states with high accuracy. However, since measuring 
biosignals requires the attachment of large numbers of sensors to the body, the technique brings a sense of constriction and may impede natural sleep. Moreover, apart from being difficult to automate, recognition of sleep states from biosignals requires judgment by a laboratory technician. Consequently, there is need to develop a method that allows for easy estimation of sleep state.

Evidently, sleep state can be estimated from biosignals such as respiratory rate, heart rate, number of body movements and lying posture. These biosignals can be measured relatively easily. For instance, a strong correlation has been found between heart rate and sleep state [7]. The relationship between the heartbeat and the number of body movements is also strong [8], [9]. Additionally, respiratory disorders such as sleep apnea syndrome are known to be unlikely to manifest when sleeping in a lateral position [10]. Watanabe et al. used air pressure mattress-sensors [11] to measure heart rate, respiration, body motion, snoring and coughing and further developed a method for estimating sleep state based on rhythm irregularities such as increased respiration and heartbeat during REM sleep [12]. Other studies use infrared motion sensors [13] or cameras [14] and focus on the extent and frequency of the measured body motion to assess the depth of sleep.

We propose a method that uses a flexible tactile sensor sheet to measure lying posture [15], respiration and heartbeat [16]. This involves the use of rubber, flexible capacitive tactile sensors that we developed (Smart Rubber (SR) sensors [17]) to measure lying posture, respiration and the heartbeat of people lying on their side in bed and in a non-restrictive manner. First, we identify lying posture based on the tactile image formed by the body pressure distribution pattern. We then obtain the rate respiration and heartbeat by measuring the pressure of the body locations in line with the recognition results. Further, lying posture, respiration and heartbeat can be measured simultaneously by using high spatial and pressure resolution SR sensors and the measured pressure information appropriately. The method has additional benefits in that, at a few $\mathrm{mm}$, it is a great deal thinner than an air mattress. Further, measurements can be performed while the subject's privacy is taken into consideration, which is different from when cameras are used. SR sensors are easy to use on a daily basis as they are cheaper than existing measuring devices and require no effort to install.

We want to estimate sleep state based on lying posture, respiration and heartbeat that have been measured using
SR sensors. However, the main challenge is that these biosignals can occasionally be inaccurately measured. For instance, heart rate causes minimal pressure fluctuations. Therefore, it is likely to be hidden by respiratory and body motion fluctuations and noise, and simultaneously difficult to measure. Moreover, errors in identifying a lying posture may also occur following the differences in physique and ways of sleeping.

Errors are highly likely in recognizing lying postures as well as in measuring the respiration and heart rates our method uses information on pressure in the locations that conform to the type of lying posture. On the other hand, if the signal intensity for the measured respiration and heartbeat is high, then it can be assumed that the recognition of the lying posture is successful. In the current paper, we propose a method that can help improve the recognition of lying posture using measured respiration and heartbeat signal intensity. First, we confirm that the success or failure of the recognition of a lying posture in the method we previously developed are correlated with the success or failure of the measurement of a heartbeat. In addition to the probability obtained through machine learning from tactile images, we use signal intensity for respiration and heartbeat to propose a method to improve the performance of the recognition of a lying posture. We demonstrate the usefulness of the proposed method by conducting an experiment using real data. In conclusion, we summarize our findings.

\section{Lying Posture Recognition Method Using Tactile Images and Respiration and Heartbeat Signal Intensity}

\subsection{Respiration and heartbeat measuring method}

First, we describe the method to measure a person's respiration and heartbeat based on the pressure data that have been measured using a flexible tactile sensor sheet (Fig. 1) [16]. We first measure the body pressure of a person lying on their side using tactile sensors laid out on the bed. We then conduct a frequency analysis for the time series data for the body pressure measured by the sensor cells in appropriate locations such as the chest etc. We used a fast Fourier transform (FFT) for the frequency analysis [18]. We obtained the person's respiration and heartbeat by extracting the appropriate frequency band.

The person whose measurements were taken was resting on a bed. While resting, respiration and heartbeat are kept virtually constant. The frequency bands for respiration and heartbeat can be separated using a band pass filter as they do not overlap in rest. In a preliminary experiment, the extracted respiration and heartbeat frequency band are 
determined as follows. (1) The respiration frequency band was set at $0.1[\mathrm{~Hz}]-0.4[\mathrm{~Hz}]$. (2) The heart rate frequency band was set at $0.8[\mathrm{~Hz}] 1.6[\mathrm{~Hz}]$. Respiration and heartbeat wave forms can be obtained by returning the frequency band to the signal of the time band. Moreover, the dominant frequency in the corresponding band after FFT should be obtained in order to obtain the respiration and heart rates alone.

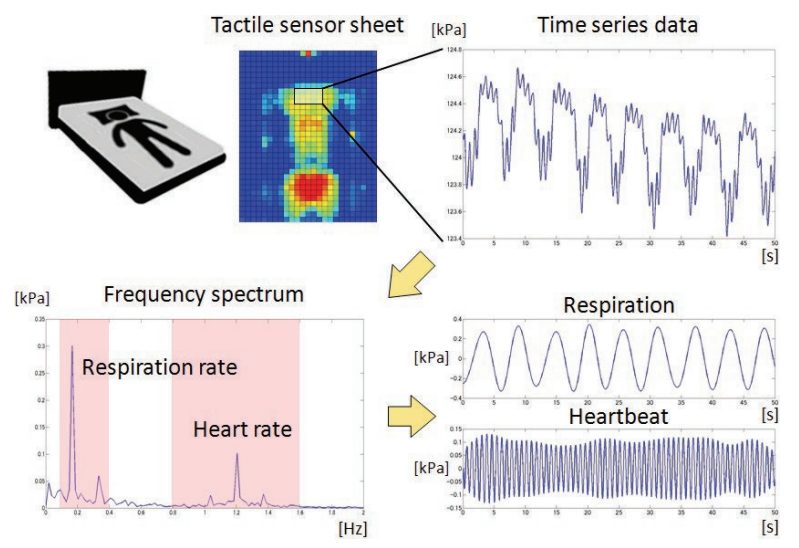

Fig. 1. Measurement method of respiration and heartbeat using a flexible tactile sensor sheet.

Furthermore, body pressure at the appropriate locations such as the chest must be measured in order to accurately measure respiration and heartbeat. A lying posture is detected using machine learning AdaBoost [19] while body pressure is measured in locations in accordance to the results (Fig. 2). This achieved robust measurements of respiration and heart rate in relation to changes in lying posture [15]. Fig. 2 shows the appropriate measuring locations where heartbeat peaks were seen in all of the 11 subjects that we took actual measurements for. For the current study, we used body pressure data of the locations where pressure was the highest, in the range shown in the ellipse in Fig. 2.
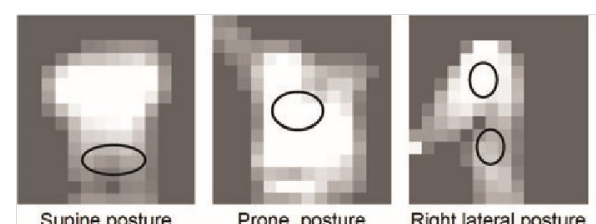

Right lateral posture

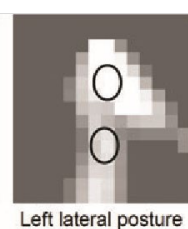

Fig. 2. Suitable locations for measurement of heartbeat. The circles show the suitable locations for different body postures. The suitable locations depend on not only the location but also the lying posture of the person [17].
Pressure fluctuations are higher for respiration than for heartbeat. Therefore, respiration can often be measured anywhere on the body and without any challenges. However, if lying posture recognition fails substantially and the pressure measuring location is placed outside the body, then it is patent that respiration cannot be measured. Fig. 3 illustrates such a case. The actual left side lying posture was erroneously detected as a supine posture. Therefore, the pressure measuring location is placed outside the body and the respiration measurement failed. The time series data shown in the top right corner of Fig. 3 reveal that the fluctuation range is extremely small and represents noise. Therefore, no peak respiration can be obtained by applying frequency analysis.

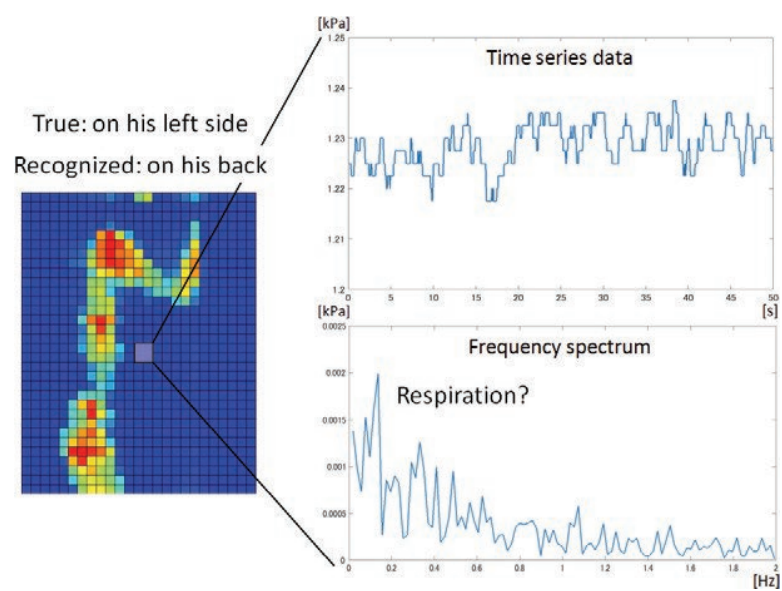

Fig. 3. Failure example of respiration measurement. We cannot obtain respiration by means of using the time series data measured at cells without body pressure.

\subsection{Improving Lying Posture Recognition Using Respiration and Heartbeat Signal Intensity}

Measuring respiration and heartbeat is more likely to fail if lying posture recognition fails. On the other hand, lying posture recognition is highly likely to be successful if signal intensity for the measured respiration and heartbeat is high. We therefore propose a method for the performance of improving lying posture recognition by using respiration and heartbeat signal intensity in addition to probability obtained from the tactile images. In specific terms, lying posture is obtained based on probability $q_{i}(\boldsymbol{x})$ in addition to probability $p_{i}(\boldsymbol{x})$ for each lying posture that is obtained through machine

learning from time series data $\boldsymbol{x}$ for the tactile images

$$
q_{i}(\boldsymbol{x})=\alpha \cdot r_{i}(\boldsymbol{x})+\beta \cdot h_{i}(\boldsymbol{x})+\gamma \cdot p_{i}(\boldsymbol{x})
$$


using respiration signal intensity $r_{i}(\boldsymbol{x})$ and heartbeat signal intensity $h_{i}(\boldsymbol{x})$ measured for each assumed lying posture. However, $\alpha, \beta, \gamma$ are weights. Eventually, they shall be $\alpha+$ $\beta+\gamma=1$ and $0 \leq \alpha, \beta, \gamma \leq 1$. Moreover, $i$ is the number of lying posture. Further, when the lying posture is category $N$, probability from $q_{1}(\boldsymbol{x})$ to $q_{N}(\boldsymbol{x})$ can be obtained. In the current study, these were the maximum lying posture recognition results. Seemingly, $r_{i}(\boldsymbol{x})$ and $h_{i}(\boldsymbol{x})$, which represent signal intensity for respiration and heartbeat, are the SN ratio. This study's experiment used, SN ratios for respiration and heartbeat, respectively.

SN ratio for respiration $S N_{r i}$ and $\mathrm{SN}$ ratio for heartbeat $S$ $N_{h i}$ are obtained based on the frequency analysis of time series data for body pressure locations in line with each of the assumed lying postures. These $\mathrm{SN}$ ratios were defined as:

$$
\begin{array}{rr}
S N_{r i}= & S \log _{10} \frac{S r i}{N_{i}}, \\
S N_{h i}= & S \log _{10} \frac{-}{N_{i}} .
\end{array}
$$

$S_{r i}, S_{h i}$, and $N_{i}$ represent the respiration, heartbeat, and noise signals respectively. The maximum spectra in the frequency bands for respiration $(0.1[\mathrm{~Hz}]-0.4[\mathrm{~Hz}])$ and heartbeat $(0.8[\mathrm{~Hz}]-1.6[\mathrm{~Hz}])$ were set to $S_{r i}$ and $S_{h i}$, respectively. Moreover, $N_{i}$ was set to the RMS of the signal in the part remaining after deducting the respiration, heartbeat and body movement signal bands from the overall frequency band. The respiration signal band is the range within $0.05[\mathrm{~Hz}]$ from frequency $f_{r i}$ to $S_{r i}$ and the second harmonic $2 f_{r i}$ and the third harmonic $3 f_{r i}$ for respiration. The heartbeat signal band is the range within $0.05[\mathrm{~Hz}]$ from frequency $f_{h i}$ to $S_{h i}$ and the second harmonic $2 f_{h i}$ for heartbeat. Further, we used a range of \pm $0.05[\mathrm{~Hz}]$ from the peak as the signal band as the peak width of the signals for respiration and heartbeat in the preliminary experiment was $0.1[\mathrm{~Hz}]$. Moreover, we set the body movement band as $0[\mathrm{~Hz}]-0.1[\mathrm{~Hz}]$.

\section{Flexible Tactile Sensors}

Sensors that are capable of high-resolution measurements of the body pressure of a person lying on a bed, in spatial terms as well as in terms of pressure are required in order to implement the proposed method. We used SR sensors [19], which are tactile sheet sensors. SR sensors, including the wiring, are entirely made of rubber. This makes them flexible and pliable and allow for measurements that avoid any sense of discomfort. Further, covering them with a sheet for instance does not result in any issues as it is possible to take measurements without the need to directly touch them.

This means that using SR sensors that are laid out on a bed allow for measurements that are non-restrictive, noninvasive and that can be conducted in circumstances that are similar to natural sleep.

SR sensors consist of a thin dielectric layer between two conductive rubber sheets of electrodes (Fig. 4). The black part of the rubber sheet are electrodes made from conductive rubber that was made using carbon filler, and are further laid out perpendicular to each other and on two layers. The total number consists of $m \times n$ cells when the number of electrodes on both sides is $m$ and $n$. This is because capacitive sensors occur where sensors on both sides intersect. Spatially high-resolution measurements are achieved by narrowing the electrodes. Further, high resolution measurements in terms of pressure can be achieved by increasing the cell surface area.

When pressure is applied, the dielectric layer deforms hence reducing the distance between the electrodes. This increases the capacitance of the said area. Scanning the capacitance of the respective cells using the sensor controller allows for the recognition of the $2 \mathrm{D}$ pressure pattern. In specific terms, a sinusoid voltage is applied to each cell. Impedance is further obtained from the current going through thus obtaining capacitance. The effect of different wire resistance values for each cell is eliminated because for equivalent circuits at the time of scanning, wire resistance is connected in a series of cell condensers. This is a sensor composition that has been proposed in the past. Further, sensors with metal electrodes are commercially available. However, for applications in care aids such as sleep monitoring systems, issues such as lack of pliability and high cost remained. We therefore made use of rubber printing technology to develop flexible and low cost SR sensors. These sensors are sold by Sumitomo Riko Co., Ltd under the name SR Soft Vision.

Table 1 outlines the specification of the SR sensors used in this study. This electrode width allows for the measurement of body pressure with sufficient spatial resolution to enable the recognition of different lying postures. On the other hand, reducing the cell area would reduce the resolution in terms of body pressure. These sensors can measure respiration without any challenges as changes in body pressure are substantial. However, (C) The 2021 International Conference on Artificial Life and Robotics (ICAROB2021), January 21 to 24, 2021 
because these changes are small in relation to heartbeat, respiration cannot be measured as is, due to noise.

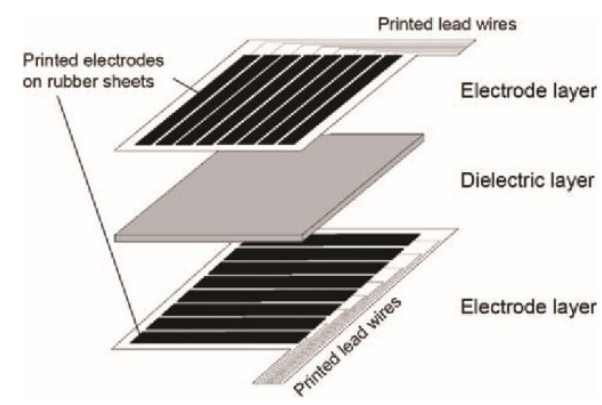

Fig. 4. Schematic structure of SR sensor.

Noise must therefore be suppressed and resolution in terms of pressure increased so as to enable the measurement of heartbeat.

Table 1. Specifications of the SR sensor.

\begin{tabular}{c|c|c}
\hline \multirow{2}{*}{ Number of cells } & \multicolumn{2}{|c}{800} \\
\cline { 2 - 3 } & Length & Width \\
\cline { 2 - 3 } Size of sensor sheet & 32 & \multicolumn{1}{c}{25} \\
\hline Size of a sensor cell & $\begin{array}{l}82 \\
{[\mathrm{~mm}]}\end{array}$ & $\begin{array}{l}686 \\
{[\mathrm{~mm}]}\end{array}$ \\
\hline Size of a gap between cells & 14 & 14 \\
& {$[\mathrm{~mm}]$} & {$[\mathrm{mm}]$} \\
\hline Thickness of sensor sheet & \multicolumn{2}{|c}{$3.5[\mathrm{~mm}]$} \\
\hline Sampling rate of all the 800 & \multicolumn{2}{|c}{$5[\mathrm{~Hz}]$} \\
cells & \multicolumn{2}{|c}{$20[\mathrm{~Hz}]$} \\
\hline $\begin{array}{c}\text { Sampling rate of the } 4 \\
\text { precision cells }\end{array}$ & \multicolumn{2}{|c}{} \\
\hline
\end{tabular}

\section{Lying Posture Recognition Experiment}

\subsection{Experimental method}

To verify the usefulness of the proposed method, we conducted an experiment to detect the lying posture of a person lying on their side on top of SR sensors laid out on a bed. The subject's positions were divided into 4 namely facing up, facing down, lying on their right side and lying on their left side. Each of these lying postures were taken 10 times. Further, time series data for body pressure were obtained for a total of 40 times. For each of these 40 data sets, probability $q_{i}(\boldsymbol{x})$, as defined in Section II-B, was obtained and lying postures detected.

First, we used the method by Mukai et al. [17] to obtain probability $p_{i}(\boldsymbol{x})$ for each lying posture based on the body pressure data. The length of one body pressure data set was set to 51.2 seconds $(51.2 \times 20[\mathrm{~Hz}]=1024)$ because conducting FFT requires that the number of data sets be a power of 2. The subject maintains the same lying posture for the full 51.2 seconds. Therefore, the obtained probability is virtually constant. The average for the 51.2 seconds was therefore set as $p_{i}(\boldsymbol{x})$. The 4 high-accuracy cells were then placed in the respective suitable locations for measuring the heartbeat for all lying postures, as shown in Fig. 2. Further, the signal intensity for respiration and heartbeat $\left(r_{i}(\boldsymbol{x})\right.$ and $\left.h_{i}(\boldsymbol{x}): i=1,2,3,4\right)$ was obtained from the time series data for body pressure. We used the $\mathrm{SN}$ ratio as the signal intensity for this study. Finally, $q_{i}(\boldsymbol{x})$ is calculated from these and lying posture recognition is performed. The present study was approved by the Research Ethics Committee of the Kyushu Institute of Technology. Further, Informed Consent was obtained in a writing from each subject taking part in the experiment.

If the signal intensity for respiration and heartbeat during the true lying posture is stronger than others, the final recognition result can be accurately corrected based on $q_{i}(\boldsymbol{x})$ by setting the appropriate weight. This can be done despite an error existing in the posture recognition using probability $p_{i}(\boldsymbol{x})$ from the tactile image only. We further conducted the following experiment to evaluate the performance of the proposed method.

Forty data sets were obtained for one subject, a 34yearold male. These data sets were labeled A1. Recognition rates were examined for various weights $(\alpha, \beta, \gamma)$ for data set A1. A confusion matrix of the 4 lying postures was also studied.

\subsection{Experimental results and discussion}

Fig. 6 shows the recognition rates with weights varying between 0 and 1 at 0.1 intervals. The horizontal and vertical axes represent weight $\alpha$ and $\beta$ respectively. The colors represent the recognition rates corresponding to the weight indicated by the bottom left coordinates. In conditions where $\alpha+\beta+\gamma=1$ and $0 \leq \alpha, \beta, \gamma \leq 1$, values with the respective weights are $\alpha=0,0.1, \cdots, 1, \beta=0,0$. $1, \cdots, 1-\alpha, \gamma=1-\alpha-\beta$. Fig. 6 does not display weight $\gamma$ as $\gamma$ is determined when $\alpha$ and $\beta$ are decided upon. 


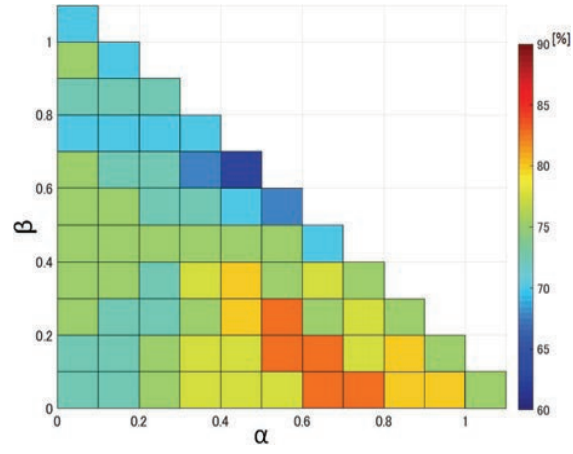

Fig. 6. Recognition rates of the lying postures (Dataset A1). Recognition of lying postures is improved by means of using a measured signal intensity of respiration and heartbeat. For example, the recognition rate of $(\alpha, \beta, \gamma)=$ $(0.6,0.1,0.3)$ is higher than that of $(\alpha, \beta, \gamma)=(0,0,1)$.

Fig. 6 shows the recognition rate is the highest at $82.5 \%$ when $(\alpha, \beta, \gamma)=(0.6,0.1,0.3)$ or close to that. In detail, weights are the highest for $(\alpha, \beta, \gamma)=(0.5,0.1,0.4)$, $(0.5,0.2,0.3),(0.6,0,0.4),(0.6,0.1,0.3),(0.7,0,0.3)$.

The recognition rate is $72.5 \%$ when $(\alpha, \beta, \gamma)=(0,0,1)$. The recognition rate for the proposed method with weight set at e.g. $(\alpha, \beta, \gamma)=(0.6,0.1,0.3)$, is $10 \%$ higher than for conventional methods that use only tactile images.

Moreover, Table 2 shows the averages and standard errors for the parameters obtained from the 40 data sets rather referred to as $\mathrm{A} 1$. These are $p_{i}(\boldsymbol{x}), r_{i}(\boldsymbol{x}), h_{i}(\boldsymbol{x}), q_{i}(\boldsymbol{x})$ when weight is $(\alpha, \beta, \gamma)=(0.6,0.1,0.3)$, with the top and bottom rows showing the parameters when lying posture is true and false respectively. A false lying posture is the posture with the highest value out of the 3 false postures. All the parameters are higher for the true lying positions. Further, it is clear that they are appropriate to use for recognition. Furthermore, the ratios for $p_{i}(\boldsymbol{x})$ and $q_{i}(\boldsymbol{x})$ for true and false lying postures are shown in rows 7 and 8 , respectively. The higher the ratio, the higher the probability for the true lying posture in relation to the probability for other lying postures. Furthermore, stable recognition is possible. We further performed a paired ttest with a significance level of 0.05 in order to examine and confirm whether there is a difference between these two ratios. The null hypothesis states that the ratio between $p_{i}(\boldsymbol{x})$ and $q_{i}(\boldsymbol{x})$ does not differ. Furthermore, the degree of freedom is 39 . This revealed that t-value $t=$ $-3.27, p=2.3 \times 10^{-3}$, and that there was no significant difference. This confirmed that in addition to the probability obtained from the tactile image, lying posture recognition can be improved by the appropriate use of signal intensity for respiration and heartbeat.

Table 2. Parameters of dataset A1 with $(\alpha, \beta, \gamma)=$ $(0.6,0.1,0.3): p_{i}(\boldsymbol{x}), q_{i}(\boldsymbol{x}), p_{\mathrm{T}}(\boldsymbol{x}) / p_{\mathrm{F}}(\boldsymbol{x})$, and $q_{\mathrm{T}}(\boldsymbol{x}) / q_{\mathrm{F}}(\boldsymbol{x})$.

Recognition performance using $q_{i}(\boldsymbol{x})$ is improved than that using $p i(\boldsymbol{x})$.

\begin{tabular}{c|c|c|c|c}
\hline $\begin{array}{c}\text { Lying } \\
\text { posture }\end{array}$ & $p_{i}(\boldsymbol{x})$ & $q_{i}(\boldsymbol{x})$ & $p_{\mathrm{T}}(\boldsymbol{x}) / p_{\mathrm{F}}(\boldsymbol{x})$ & $q_{\mathrm{T}}(\boldsymbol{x}) / q_{\mathrm{F}}(\boldsymbol{x})$ \\
\hline True & $\begin{array}{c}50.1 \pm \\
15.7\end{array}$ & $\begin{array}{c}40.2 \pm \\
5.8\end{array}$ & $\begin{array}{c}2.09 \pm \\
1.30\end{array}$ & $\begin{array}{c}2.42 \pm \\
1.04\end{array}$ \\
\hline False & 29.719 .0 & 19.811 .6 & & \\
\hline
\end{tabular}

\section{Conclusion}

We proposed a method to improve the performance of lying posture recognition by using measured respiration and heartbeat signal intensity. We conducted experiments using actual data and demonstrated the fact that recognition performance can be improved by using respiration and heartbeat signal intensity along with the probability obtained from the tactile images. Moreover, we used data from different trials with the same subject and data for different subjects to confirm the versatility of the proposed method.

\section{References}

[1] Statistics Bureau, Ministry of Internal Affairs and Communications, Japan. (2011, Oct.). The 2010 Population Census of Japan. Public announcement on 26 Oct. 2011. [Online]. Available: $\quad<$ http://www. stat.go.jp/english/data/kokusei/index.htm>, (accessed on 3rd Dec. 2020)

[2] National Institute of Population and Social Security Research. (2012, Mar.). Population Projections for Japan (January 2012) 2011 to 2060-. Public announcement on 30 Mar. 2012. [Online]. Available: $<$ http://www.ipss.go.jp/site$\mathrm{ad} /$ index _ english/ esuikei/gh2401e.asp>, (accessed on 3rd Dec. 2020)

[3] Ministry of Health, Labour and Welfare, Japan. (2011, June). General Welfare and Labour. Public announcement on 2 June 2011. [Online]. Available: $<$ http://www.mhlw.go.jp/english/policy/ other/socialsecurity/index.html $>$, (accessed on 3rd Dec.2020).

[4] Ministry of Economy, Trade and Industry and Ministry of Health, Labor and Welfare, Japan. (2012, Nov.). Priority Areas to Which Robot

Technology Is to Be Introduced in Nursing Care of the Elderly. Public announcement on 22 Nov. 2012. [Online]. Available:

$<$ http://robotcare.jp/en/home/index.php?\\&lang=en $\backslash \&$ PHPS 
$\mathrm{ESSID}=\mathrm{q} 34 \mathrm{fa} 48 \mathrm{pe} 9 \mathrm{plr3go0o0}$ vndmon $>$, (accessed on 3rd Dec. 2020).

[5] C. A. Kushida, M. R. Littner, T. Morgenthaler, C. A. Alessi, D. Bailey,

J. Coleman Jr, L. Friedman, M. Hirshkowitz, S. Kapen, M. Kramer,

T. Lee-Chiong, D. L. Loube, J. Owens, J. P. Pancer, and M. Wise, "Practice parameters for the indications for polysomnography and related procedures: An update for 2005," Journal of Sleep, vol. 28, no. 4, pp. 499-521, 2005.

[6] A. Rechtschaffen and A. Kales, A Manual of Standardized Terminology, Techniques and Scoring System for Sleep Stages of Human Subjects. Bethesda, MD. : U.S. Dept. of Health, Education, and Welfare, Public Health ServicesNational Institutes of Health, National Institute of Neurological Diseases and Blindness, Neurological Information Network, 1968.

[7] T. Watanabe and K. Watanabe, "Estimation of the Sleep Stages by the Non-restrictive Air Mattress Sensor: Relation bitween the Change in the Heart Rate and Sleep Stages," Trans. of the Society of Instrument and Control Engineers, vol. 37, no. 9, pp. 821-828, 2001.

[8] A. Deguchi and H. Hagiwara, "Evaluation of Heart Rate Variability and Respiratory Variability during Sleep Using a Lorenz Plot," Journal of Sleep and Biological Rhythms, vol. 9, no. 4, p. 366, 2011.

[9] K. Otsuka, Y. Ichimaru, T. Yanaga, and Y. Sato, "Studies of Arrhythmias by 24-hour Polygraphic Recordings: Relationship between Atrioventricular Block and Sleep States", American Heart Journal, vol. 105, no. 6, pp. 934 $940,1983$.

[10] Z. Beattie, C. Hagen, and T. Hayes, "Classification of Lying Position Using Load Cells under the Bed", in Proc. 33rd Annual Int'l Conf. of the IEEE Engineering in Medicine and Biology Society, pp. 474-477, 2011.

[11] Y. Kurihara and K. Watanabe, "Sleep-Stage Decision Algorithm by Using Heartbeat and Body-Movement Signals," IEEE Trans. on Systems, Man, and Cybernetics, Part A, vol. 42, no. 6, pp. 1450-1459, 2012.

[12] T. Watanabe and K. Watanabe, "Noncontact Method for Sleep Stage Estimation," IEEE Trans. on Biomedical Engineering, vol. 51, no. 10, pp. 1735-1748, 2004.

[13] Y. Kambayashi and H. Hagiwara, "Estimating Sleep Cycle Using Body Movement Density," in Proc. 2012 5th Int'l Conf. on Biomedical Engineering and Informatics, pp. 1029-1033, 2012.

[14] S. Okada, Y. Ohno, Goyahan, K. Kato-Nishimura, I. Mohri, and M. Taniike, "Examination of Non-restrictive and Noninvasive Sleep Evaluation Technique for Children Using Difference Images," in Proc. 30th Annual Int'l Conf. of the IEEE Engineering in Medicine and Biology Society, pp. 2483-2487, 2008.

[15] T. Mukai, K. Matsuo, Y. Kato, A. Shimizu, and S. Guo, "Lying Posture Detection for Unconstrained Measurement of Respiration and Heartbeat of a Person on a Bed," in Proc. 11th IASTED Int'l Conf. on Biomedical Engineering, pp. 46-53, DOI: 10.2316/P.2014.818-014, 2014.
[16] K. Matsuo, T. Mukai, Y. Kato, A. Shimizu, and S. Guo, "Measurement of Respiration and Heartbeat Using a Flexible Tactile Sensor Sheet on a Bed," in Proc. 11th IASTED Int'l Conf. on Biomedical Engineering, pp. 59-64, DOI: 10.2316/P.2014.818-031, 2014.

[17] S. Guo, Y. Kato, H. Ito, and T. Mukai, "Development of Rubber-Based Flexible Sensor Sheet for Care-Related Apparatus," Sumitomo Electric Industries Technical Review, no. 75 , pp. 125-131, 2012.

[18] J. W. Cooley and J. W. Tukey, "An Algorithm for the Machine Calculation of Complex Fourier Series," Mathematics of Computation, vol. 19, no. 90, pp. 297-301, 1965.

[19] Y. Freund and R. Schapire, "A Decision-Theoretic Generalization of On-Line Learning and an Application to Boosting", Journal of Computer and System Science, vol. 55, pp. 119-139, 1997.

[20] 